\title{
Effects of semantic context in the naming of pictures and words
}

\author{
Markus F. Damian*, Gabriella Vigliocco, Willem J.M. Levelt \\ Max Planck Institute for Psycholinguistics, Nijmegen, The Netherlands
}

Received 16 June 2000; received in revised form 10 February 2001; accepted 10 April 2001

\begin{abstract}
Two experiments investigated whether lexical retrieval for speaking can be characterized as a competitive process by assessing the effects of semantic context on picture and word naming in German. In Experiment 1 we demonstrated that pictures are named slower in the context of same-category items than in the context of items from various semantic categories, replicating findings by Kroll and Stewart (Journal of Memory and Language, 33 (1994) 149). In Experiment 2 we used words instead of pictures. Participants either named the words in the context of same- or different-category items, or produced the words together with their corresponding determiner. While in the former condition words were named faster in the context of samecategory items than of different-category items, the opposite pattern was obtained for the latter condition. These findings confirm the claim that the interfering effect of semantic context reflects competition in the retrieval of lexical entries in speaking. (C) 2001 Elsevier Science B.V. All rights reserved.
\end{abstract}

Keywords: Semantic context; Naming; Pictures and words

\section{Introduction}

In speech production tasks such as picture naming, semantic similarity exerts a predominantly interfering influence. For instance, in picture-word interference tasks, a semantic relationship between a target picture and a distractor slows

\footnotetext{
* Corresponding author. Present address: Department of Experimental Psychology, University of Bristol, 8 Woodland Road, Bristol BS8 1TN, UK. Fax: +44-117-928-8588.

E-mail address: m.damian@bristol.ac.uk (M.F. Damian).
}

0010-0277/01/\$ - see front matter (C) 2001 Elsevier Science B.V. All rights reserved.

PII: S0010-0277(01)00135-4 
responses relative to an unrelated condition (e.g. Glaser \& Düngelhoff, 1984; Lupker \& Katz, 1981; Schriefers, Meyer, \& Levelt, 1990).

Such interference can be accounted for in terms of competition among co-activated lexical entries by virtue of their semantic relatedness. This account will be termed the "lexical competition hypothesis". For instance, Roelofs (1992) assumes that conceptual input from the picture forms a cohort of related, mutually activating lexical concepts, each of which connects to a lemma which represents the word's syntax. Hence, when lexical concepts are co-activated, so are their lemmas. The activation is, moreover, two-way (Levelt, Roelofs, \& Meyer, 1999). Co-activated lemmas compete for retrieval. Selecting the target lemma is thus delayed by the simultaneous processing of a related item. Such a principle of lexical competition is also implemented in other (e.g. Caramazza, 1997; Humphreys, Riddoch, \& Quinlan, 1988), but not in all (e.g. Dell, 1986; MacKay, 1987) models of speaking.

Interfering effects of semantic context were also reported by Kroll and Stewart (1994) in picture naming and word translation. In one experiment, participants named pictures from various semantic categories in lists that were either blocked or randomized by semantic categories. Latencies for an item within the context of the same category were slower than within the context of other categories, a finding that was also obtained in single word translation. These finding were taken to support the lexical competition assumption.

To allow for this conclusion, other potential factors need to be excluded. On the one hand, the effect may arise because objects from a particular semantic category often look similar, and thus, visual confusability in the blocked condition might slow responses. ${ }^{1}$ On the other hand, semantic context might generate a non-linguistic conceptual conflict due to overlapping representations, in which case the effect would not reflect language production processes. The first goal of the research reported here is to address these potential confounds. The second goal is to assess the lexical nature of the competition effect from a different angle. According to a number of authors, lemmas specify syntactic (Levelt et al., 1999) or semantic and syntactic properties (Vigliocco, Vinson, Martin, \& Garrett, 1999). Grammatical gender of German nouns, therefore, is specified at this level. When German speakers produce "determiner + noun" phrases, given the printed noun, they must select the noun's lemma to select the appropriated gender-marked determiner. If retrieval at this level is a competitive process, we should then observe interference in this task. Competition, instead, should not arise when speakers are simply asked to read aloud the printed word since this can be carried out at the form level to a large extent (e.g. Morton \& Patterson, 1980).

Our experiments test these different hypotheses. Experiment 1 tackles the visual confusability account by employing a picture naming task in which the visual similarity confound has been largely reduced. The conceptual conflict account is tested in a follow-up experiment with a manual response task that does not require

\footnotetext{
${ }^{1}$ Although Kroll and Stewart (1994) obtained similar results in a word translation experiment which would not be subject to this potential confound, relatively long response latencies of approximately 1200 ms with error scores above $40 \%$ make the interpretation problematic.
} 
lexical retrieval. The lexical competition account is directly tested in Experiment 2, using a word naming task.

\section{Experiment 1}

This experiment uses the Kroll and Stewart (1994) paradigm, but controls for visual similarity across the conditions. Because for most semantic categories only a few visually distinct exemplars are available, the experiment employs a small number of exemplars per category. Each picture is named repeatedly within the context of same-category items (henceforth called "homogeneous"), or in the context of a variety of categories ("heterogeneous"). If visual similarity can be sufficiently reduced, then the effect of semantic context would be attributable to post-visual processing stages.

\subsection{Method}

\subsubsection{Participants}

Ten native German speakers from the participants pool of the Max Planck Institute of Cognitive Neuroscience in Leipzig took part in this experiment in exchange for pay.

\subsubsection{Materials}

Twenty-five line drawings of common objects were selected from five semantic categories (see Appendix A). These were arranged in a matrix of $5 \times 5$ items such that rows corresponded to categories and thus formed homogeneous stimulus sets of five items each while columns formed the sets for the heterogeneous condition of the same size.

Items were selected to minimize within-category visual similarity. By much trial and error, and guided by systematic visual similarity judgments, we could largely eliminate the tendency of within-category items to be visually similar. In a final rating $(N=20)$, participants were instructed to ignore semantic similarity and instead to judge the visual similarity of all 100 pairwise picture combinations on a scale ranging from 1 (not at all similar) to 5 (very similar). As compared to an average between-category similarity of 1.97 , the within-category similarity was only slightly higher at 2.45 . Choosing maximally dissimilar within-category items had as a consequence that overlapping initial phonemes within a picture set could not entirely be avoided. Instead, homogeneous and heterogeneous sets were matched such that in each condition, four of the five sets contained one stimulus pair that shared the initial segment. ${ }^{2}$

\footnotetext{
${ }^{2}$ To further assess phonological overlap, a similarity score (see Damian \& Martin, 1999) was computed for all pairwise stimulus combinations. The resulting average scores were 0.106 and 0.090 for the homogeneous (same-category) and heterogeneous (different-category) conditions, respectively. The two conditions were thus closely matched with regard to their phonological characteristics.
} 


\subsubsection{Design}

Semantic context (homogeneous vs. heterogeneous) was manipulated as a withinsubjects variable. Twenty experimental blocks (ten homogeneous and ten heterogeneous blocks) were presented in an ABBA design such that five consecutive blocks were of the same type. Within each block, each of the five target pictures was presented five times in a pseudorandom order such that the same picture never appeared on consecutive trials. Each testing session lasted about $40 \mathrm{~min}$.

\subsubsection{Procedure}

Stimuli (size: approximately $7 \times 7 \mathrm{~cm}$ ) were presented on a computer monitor. Responses were measured with a voice trigger. In a practice block, each picture was presented and named once in random order. Then, the 20 experimental blocks were carried out.

On each trial, a fixation cross was presented for $500 \mathrm{~ms}$. After a blank period of $500 \mathrm{~ms}$, the target picture was shown for $500 \mathrm{~ms}$. Latencies were measured from the onset of the target. An intertrial interval of 1500 ms concluded each trial.

\subsection{Results}

Latencies on error trials, and latencies larger than $1500 \mathrm{~ms}$ or smaller than $200 \mathrm{~ms}$, were excluded. As context effects are being targeted, the first occurrence of each stimulus on each block was also excluded.

Mean response latencies were $567 \mathrm{~ms}$ for the heterogeneous condition, and 596 $\mathrm{ms}$ for the homogeneous condition. An ANOVA showed the difference of $29 \mathrm{~ms}$ to be highly significant $(F 1(1,9)=10.79, \quad \mathrm{MSE}=3955, \quad P=0.009$; $F 2(1,24)=23.49$, MSE $=9887, P<0.001)$. A similar pattern was found in the error scores, with a mean score of $0.6 \%$ for the heterogeneous condition, and one of $2.0 \%$ for the homogeneous condition. This difference was significant $(F 1(1,9)=10.14, \quad \mathrm{MSE}=9.80, \quad P=0.011 ; \quad F 2(1,24)=7.06, \quad \mathrm{MSE}=24.50$, $P=0.014)$.

\subsection{Discussion}

We found a competition effect even when visual similarity in the homogeneous condition was reduced to a minimum. These findings replicate the results of Kroll and Stewart (1994) and are compatible with the lexical competition account. However, the possibility remains that the effect arises pre-lexically, caused by conceptual competition within a homogeneous category, which would predict that it would still arise in a conceptually mediated task that does not require lexicalization. The lexical competition account predicts that the effect should disappear in such a task. ${ }^{3}$ The pictures used in Experiment 1 had been selected such that symmetrical objects or perspectives were avoided, but each object faced in a particular direction. For instance, the picture of a SHOE faces towards its tip, while the picture

\footnotetext{
${ }^{3}$ A similar logic was applied in Schriefers et al. (1990, Experiment 3) and Jescheniak and Levelt (1994, Experiment 2).
} 
of a DUCK faces towards its head. A judgment with regard to the direction in which an object faces requires access to conceptual knowledge, as it cannot be made on the basis of visual characteristics such as protruding edges.

The results of this experiment $(N=10)$ showed a mean of $396 \mathrm{~ms}$ for the heterogeneous condition, and a mean of $388 \mathrm{~ms}$ for the homogeneous condition. Thus, the semantic interference effect obtained in Experiment 1 was eliminated. But with any bimanual task and multiple presentations of a small set of stimuli, participants might have based their responses on learned stimulus-response associations rather than on genuine semantic access. In a further experiment $(N=10)$, each object faced in either direction with equal probability. The results were virtually identical, with means of $385 \mathrm{~ms}$ for the homogeneous condition and $378 \mathrm{~ms}$ for the heterogeneous condition. Thus, the semantic interference effect obtained in Experiment 1 appears to be specific to tasks that require lexical retrieval. In Experiment 2, we tested the lexical competition hypothesis directly.

\section{Experiment 2}

In two main conditions, printed nouns are presented in a semantically homogeneous or heterogeneous context. In one condition, speakers are required to produce a "determiner + noun" phrase in response to the bare word (henceforth "NP"). In languages such as German, which possess a grammatical gender system, determiners vary according to the noun's gender; thus, gender constitutes a genuine lexical variable that must be stored in the lexicon, but is neither conceptually nor phonologically conditioned. In models of speech production that assume a syntactically specified lemma, lemma retrieval is mandatory in the access of gender, and consequently, we should observe competition. In the other condition, speakers are required to produce a bare noun ("N"). Studies conducted on the reading aloud of printed words have generally shown facilitatory context effects. The standard account is that words access their semantic specification and spread activation to related items which are therefore retrieved more easily as targets on subsequent trials. However, facilitatory effects in naming are usually reduced in magnitude in comparison to parallel effects in non-naming tasks such as lexical decision (Neely, 1991), and are absent in some studies (Kroll \& Stewart, 1994). Crucially, no competition is predicted in this condition.

A predicted dissociation of semantic competition between NP (present) and N naming (absent) would strongly underscore the assumption that lexical competition generates the interference effect.

\subsection{Method}

\subsubsection{Participants}

Twenty-four native German speakers from the participants pool of the Max Planck Institute for Psycholinguistics in Nijmegen took part in this experiment in exchange for pay. Twelve participants were randomly assigned to each task group. 


\subsubsection{Materials}

Thirty-six German nouns from six semantic categories were selected, with three masculine items (determiner: der) and three feminine items (determiner: die) per category (see Appendix A). In a matrix of $6 \times 6$ items with rows corresponding to the categories, items were arranged such that columns, constituting the heterogeneous sets, included three items of each gender. None of the items within a set shared the initial phoneme or letter. ${ }^{4}$

\subsubsection{Design}

Task (NP vs. N) was manipulated as a between-subjects variable, and semantic context (homogeneous vs. heterogeneous) as a within-subjects variable. Six homogeneous and six heterogeneous blocks were presented. Within a block, each of the six targets was presented ten times in a pseudorandom fashion such that the same word never appeared on consecutive trials, and the same gender did not appear more than three times in sequence. Blocks were presented in an alternating sequence of homogeneous and heterogeneous blocks. The order of the blocks was determined by a sequentially balanced Latin square design. Each testing session lasted about 45 $\min$.

\subsubsection{Procedure}

Participants were instructed to name a word presented on the screen either with its appropriate determiner (NP group) or by itself ( $\mathrm{N}$ group). The apparatus was the same as in Experiment 1. Words were presented in white Arial 36 lowercase font on a black background at the center of the screen. All words were presented and named once in a practice block in random order, followed by the 12 experimental blocks.

On each trial, a fixation cross was presented for $500 \mathrm{~ms}$. After a blank period of $500 \mathrm{~ms}$, the target word was shown for $500 \mathrm{~ms}$. Latencies were measured from the onset of the target. An intertrial interval of $1500 \mathrm{~ms}$ concluded each trial.

\subsection{Results}

Errors (less than $1 \%$ of the trials) were not analyzed, but eliminated from the analysis, as well as latencies larger than $1000 \mathrm{~ms}$ or smaller than $200 \mathrm{~ms}$, and the first occurrence of each stimulus on each block.

In the NP group, response latencies were $486 \mathrm{~ms}$ in the heterogeneous condition and 497 in the homogeneous condition (an $11 \mathrm{~ms}$ interference effect). In the $\mathrm{N}$ group, response latencies were $488 \mathrm{~ms}$ in the heterogeneous condition and $475 \mathrm{~ms}$ in the homogeneous condition (a $13 \mathrm{~ms}$ facilitation effect). Fig. 1 displays the evident interaction between task and context.

An ANOVA with the factors task and semantic context indicated no main effects $(F<1)$, but a significant interaction between the two factors $(F 1(1,22)=10.85$,

\footnotetext{
${ }^{4}$ As before, a similarity index was computed both for segments and for letters. Average segmental overlap was 0.167 and 0.155 for the homogeneous and heterogeneous conditions, respectively, while letter overlap was 0.222 and 0.223 . Items in the two conditions were thus matched with regard to their form characteristics.
} 

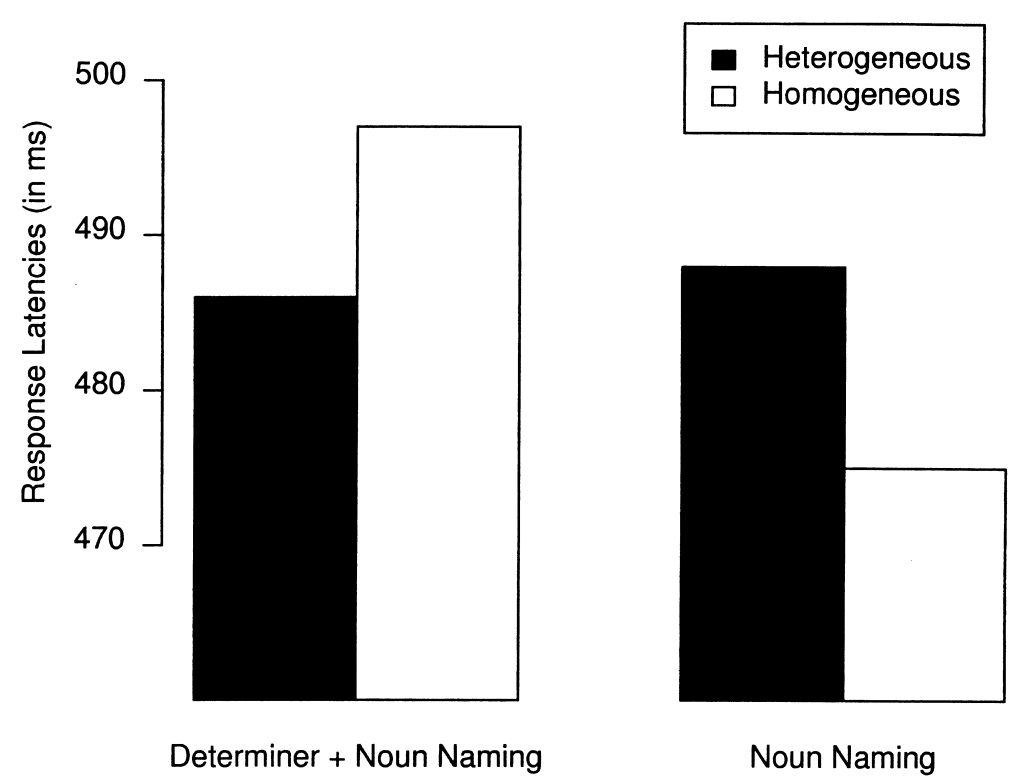

Fig. 1. Response latency means by task group (determiner + noun naming vs. noun naming) and semantic context (homogeneous vs. heterogeneous).

$\mathrm{MSE}=1697, P=0.003 ; F 2(1,35)=40.86, \mathrm{MSE}=5298, P<0.001)$. Simple effects of semantic context, performed separately for each task group, showed a significant effect in the NP group $(F 1(1,11)=5.77, \mathrm{MSE}=745, P=0.035$; $F 2(1,35)=20.39, \quad \mathrm{MSE}=2354, \quad P<0.001), \quad$ as well as in the $\mathrm{N}$ group $(F 1(1,11)=5.22, \quad \mathrm{MSE}=958, \quad P=0.043 ; \quad F 2(1,35)=24.58, \quad \mathrm{MSE}=2962$, $P<0.001)$. Thus, semantic context significantly affected responses in each task group, but in opposite directions.

\subsection{Discussion}

In the determiner + noun naming task, an interfering context effect was obtained, paralleling the one observed in Experiment 1 in picture naming. This effect was absent in bare noun naming. In fact, compatible with previous studies of semantic effects in word naming, semantic facilitation was observed. What caused the interference effect in NP naming? In bare noun naming, phonological codes can be directly accessed from the orthographic input codes, as the task does not require lexical-semantic retrieval. In our determiner + noun naming task, however, lexicalsemantic retrieval is obligatory. Producing a noun's determiner in German requires access to the noun's syntactic gender, a lemma-level property. For instance, in the framework proposed by Levelt et al. (1999), an accessed lemma spreads activation to the corresponding lexical concept, which co-activates related concepts and their lemmas. This, in turn, will lead to competition among semantically related lemmas, our observed interference effect. The finding of an interference effect in phrase 
production further falsifies the conceptual conflict account described in Section 1 because here semantic interference was obtained when the task involved the retrieval of a syntactic - language-specific - property.

The facilitation we observed in bare noun production could have been caused by interactive processes between orthographic input and semantic codes, or cascading activation from the semantic to the phonological level, but it might also be of purely strategic origin: participants surely noticed the semantic blocking and consequently might have developed expectancies or employed retrospective checking (e.g. Neely, Keefe, \& Ross, 1989). The current findings do not allow us to distinguish between these possibilities. ${ }^{5}$

\section{General discussion}

Pictures are named slower in the context of same-category items than in the context of different-category items even when within-category visual similarity is greatly reduced (Experiment 1). Such a difference is not obtained in a manual response task (follow-up experiment). In a parallel fashion, when speakers produce phrases from visually presented words, we obtain the same semantic interference effect as in picture naming, whereas in bare word naming, the semantic context effect is reversed (Experiment 2). In combination, these findings falsify accounts that attribute the semantic interference effect in this production task either to visual confusability between same-category objects or to a general conceptual conflict. Instead, they support an account in terms of competitive lexical retrieval.

In most theories of speaking since Garrett (1975), the retrieval of abstract lexical representations (i.e. lemmas) mediates between conceptual and phonological access. The current findings show that semantic similarity plays an important role, a fact that is also illustrated by the observation that for the majority of spontaneously occurring lexical speech errors, target and intruding words are semantically related (e.g. Garrett, 1980).

Overall, the finding that semantic interference can be induced by requiring speakers to produce phrases instead of single words suggests a rather close link between semantic and syntactic knowledge: retrieval of a syntactic property (e.g. gender) entails semantic consequences. For instance, in the architecture proposed by Levelt et al. (1999), co-activation of lexical concepts and lemmas comes about "top-down" in picture naming, or "bottom-up" in word naming where the gender-marked determiner has to be produced.

The semantic interference effect presented here is fully compatible with findings reported by Vitkovitch and Humphreys (1991) (see also Vitkovitch, Humphreys, \& Lloyd-Jones, 1993) that demonstrated increased error rates in speeded picture naming when targets were preceded by items from the same semantic category;

\footnotetext{
${ }^{5}$ It is also possible that the semantic facilitation effect observed in bare noun naming was counteracting the semantic interference effect observed in determiner + noun naming to some extent. This would account for the fact that numerically, the interference effect in the determiner + noun condition was smaller than the one obtained in Experiment 1.
} 
most likely the same mechanism - lexical competition - is at the root of this effect. However, in contrast to the claims derived from these studies, the current findings have no bearing on the issue of whether speech production is modular or cascaded.

\section{Acknowledgements}

We would like to thank Marc Brysbaert and Ardi Roelofs for helpful comments, and Otto Kokke and Ilse van Leiden for assistance in collecting the data. Further thanks go to Angela Friederici for making the participants pool of the Max Planck Institute of Cognitive Neuroscience in Leipzig available to us.

\section{Appendix A}

\section{A.1. Stimuli used in Experiment 1}

Mean word form frequency of the picture labels in the CELEX corpus of spoken German: 39 per million. The approximate English translation is given in brackets. ANIMALS: Maus (mouse), Spinne (spider), Schlange (snake), Fisch (fish), Ente (Duck)

VEHICLES: Zug (train), Fahrrad (bicycle), Auto (car), Flugzeug (airplane), Bus (bus)

CLOTHING: Hose (pants), Kleid (dress), Socke (sock), Weste (vest), Schuh (shoe)

TOOLS: Besen (broom), Axt (axe), Säge (saw), Bohrer (drill), Schere (scissors)

FURNITURE: Sessel (armchair), Sofa (couch), Tisch (table), Schrank (wardrobe), Lampe (lamp)

\section{A.2. Stimuli used in Experiment 2}

Mean CELEX word form frequency: 20 per million. Within each category, the first three items are of feminine grammatical gender, while the others are of masculine gender.

ANIMALS: Katze (cat), Ziege (goat), Ratte (rat), Biber (beaver), Tiger (tiger), Schwan (swan)

BODY PARTS: Hand (hand), Rippe (rib), Nase (nose), Schenkel (thigh), Mund (mouth), Arm (arm)

VEHICLES: Fähre (ferry), Kutsche (carriage), Galeere (galley), Traktor (tractor), Bus (bus), Wagen (car)

CLOTHING: Rock (skirt), Schal (scarf), Mantel (coat), Jacke (jacket), Socke (sock), Hose (pants)

TOOLS: Besen (broom), Hammer (hammer), Pinsel (paint brush), Zange (pliers), Schere (scissors), Säge (saw)

VEGETABLES: Lauch (leek), Kohl (cabbage), Salat (lettuce), Erbse (pea), Zwiebel (onion), Gurke (cucumber) 


\section{References}

Caramazza, A. (1997). How many levels of processing are there in lexical access. Cognitive Neuropsychology, 14, 177-208.

Damian, M. F., \& Martin, R. C. (1999). Semantic and phonological codes interact in single word production. Journal of Experimental Psychology: Learning, Memory, and Cognition, 25, 345-361.

Dell, G. S. (1986). A spreading activation theory of retrieval in sentence production. Psychological Review, 93, 283-321.

Garrett, M. (1975). The analysis of sentence production. In G. H. Bower, The psychology of learning and motivation (Vol. 9, pp. 133-177). 9. New York: Academic Press.

Garrett, M. (1980). The limits of accommodation: arguments for independent processing levels in sentence production. In V. A. Fromkin (Ed.), Errors in linguistic performance: slips of the tongue, ear, pen, and hand (pp. 263-271). London: Academic Press.

Glaser, W. R., \& Düngelhoff, F.-J. (1984). The time course of picture-word interference. Journal of Experimental Psychology: Human Perception and Performance, 10, 640-654.

Humphreys, G. W., Riddoch, M. J., \& Quinlan, P. T. (1988). Cascade processes in picture identification. Cognitive Neuropsychology, 5, 67-104.

Jescheniak, J. D., \& Levelt, W. J. M. (1994). Word frequency effects in speech production: retrieval of syntactic information and phonological form. Journal of Experimental Psychology: Learning, Memory, and Cognition, 20, 824-843.

Kroll, J. F., \& Stewart, E. (1994). Category interference in translation and picture naming: evidence for asymmetric connections between bilingual memory representations. Journal of Memory and Language, 33, 149-174.

Levelt, W. J. M., Roelofs, A., \& Meyer, A. S. (1999). A theory of lexical access in speech production. Behavioral and Brain Sciences, 22, 1-38.

Lupker, S. J., \& Katz, A. N. (1981). Input, decision, and response factors in picture-word interference. Journal of Experimental Psychology: Human Learning and Memory, 7, 269-282.

MacKay, D. (1987). The organization of perception and action: a theory for language and other cognitive skills. New York: Springer.

Morton, J., \& Patterson, K. E. (1980). A new attempt at an interpretation, or, an attempt at a new interpretation. In M. Coltheart, K. E. Patterson, \& J. C. Marshall (Eds.), Deep dyslexia (pp. 91118). London: Routledge and Kegan Paul.

Neely, J. H. (1991). Semantic priming effects in visual word recognition: a selective review of current findings and theories. In D. Besner \& G. W. Humphreys (Eds.), Basic processes in reading: visual word recognition (pp. 264-336). Hillsdale, NJ: Lawrence Erlbaum.

Neely, J. H., Keefe, D. E., \& Ross, K. (1989). Semantic priming in the lexical decision task: roles of prospective prime-generated expectancies and retrospective relation-checking. Journal of Experimental Psychology: Learning, Memory, and Cognition, 15, 1003-1019.

Roelofs, A. (1992). A spreading-activation theory of lemma retrieval in speaking. Cognition, 42, 107-142.

Schriefers, H., Meyer, A. S., \& Levelt, W. J. M. (1990). Exploring the time course of lexical access in language production: picture-word interference studies. Journal of Memory and Language, 29, 86102.

Vigliocco, G., Vinson, D. P., Martin, R. C., \& Garrett, M. F. (1999). Is “count" and "mass” information available when the noun is not? An investigation of tip of the tongue states and anomia. Journal of Memory and Language, 40, 534-558.

Vitkovitch, M., \& Humphreys, G. W. (1991). Perseverant responding in speeded naming of pictures: its in the links. Journal of Experimental Psychology: Learning, Memory, and Cognition, 17, 664-680.

Vitkovitch, M., Humphreys, G. W., \& Lloyd-Jones, T. J. (1993). On naming a giraffe a zebra: picture naming errors across different object categories. Journal of Experimental Psychology: Learning, Memory, and Cognition, 19, 243-259. 